\title{
Erratum: Quasielectrons in lattice Moore-Read models [Phys. Rev. B 99, 045147 (2019)]
}

\author{
Sourav Manna, Julia Wildeboer, and Anne E. B. Nielsen \\ (Received 23 October 2020; published 13 November 2020)
}

DOI: 10.1103/PhysRevB.102.199901

We correct errors due to a mistake in the Monte Carlo code in Sec. II of our paper which represent the overlap $\mathcal{O}$ and the ratio $\mathcal{N}$ of the respective norms of the two states in Fig. 3(b). We show here the corrected plot Fig. 3(b).

The conclusions of the results remain the same also with the new data. The decay factor values as mentioned after Eq. (22) in our paper are modified as follows. For the case of four quasielectrons, the decay coefficient is $\lambda=0.0263$ for $\mathcal{O}$ and $\lambda=0.0271$ for $\mathcal{N}$. For the case of two quasiholes and two quasielectrons, the decay coefficient is $\lambda=0.0416$ for $\mathcal{O}$ and $\lambda=0.0422$ for $\mathcal{N}$.

We note that these corrections do not alter the conclusions of Sec. II and, hence, the conclusions of our paper remain the same.

We thank B. Jaworowski for pointing out the mistakes which we have now corrected.

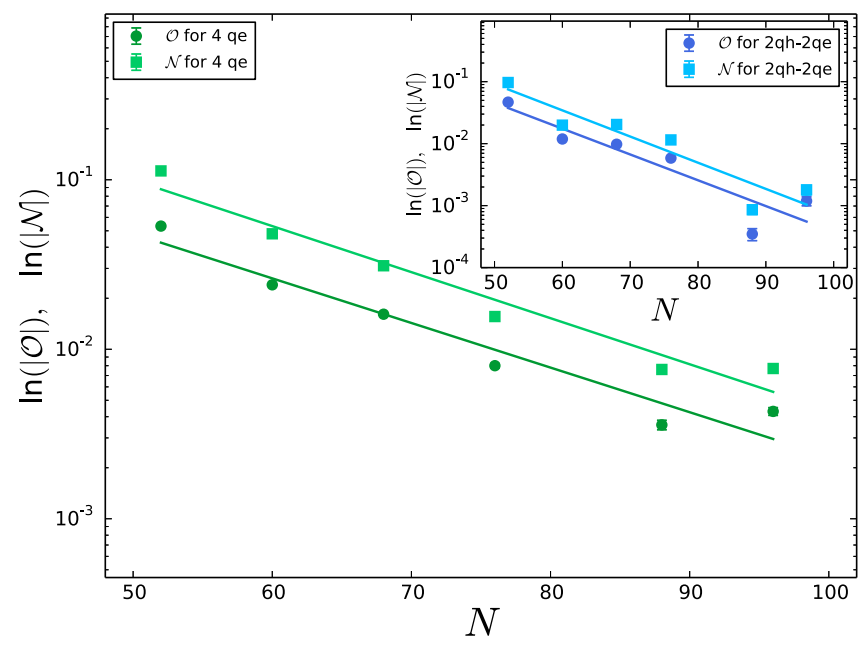

FIG. 3. We plot $\mathcal{O}$ (circles) and $\mathcal{N}$ (squares) in the semilogarithmic scale as a function of the lattice size $N$ for the cases of four quasielectrons ( $4 \mathrm{qe}$ ) and two quasiholes-two quasielectrons (2qh-2qe) present in the system. We note that the quantities of interest in both the plots are following an exponential decay. 